Chirurg 2017 $\cdot 88: 165$

DOI 10.1007/s00104-017-0370-y

Online publiziert: 19. Januar 2017

๑) Springer Medizin Verlag Berlin 2017

CrossMark

W. Schröder · C. J. Bruns

Klinik für Allgemein-, Viszeral- und Tumorchirurgie, Universitätsklinik Köln, Köln, Deutschland

\title{
Einfluss der Hiatushernie auf das postoperative Outcome nach Ösophagektomie
}

tushernie $>5 \mathrm{~cm}$ und 325 Patienten ohne oder mit Hitaushernie $<5 \mathrm{~cm}$.

Gandon A, Gronnier C, Renaud F et al (2016) Esophageal adenocarcinoma - Impact of large hiatal hernia on outcome after surgery. Ann Surg 264:862-870

Hintergrund und Fragestellung. Durch die steigende Inzidenz der Adenokarzinome des distalen Ösophagus werden auch zunehmend Patienten mit Hiatushernien operiert. Diese Begleitdiagnose, die in der Literatur mit einer Prävalenz von bis zu $20 \%$ angegeben wird, erschwert sowohl das präoperative Staging des Primärtumors als auch die intraoperative chirurgische Präparation. Insbesondere wird die präoperative Diagnose einer Hiatushernie mit der postoperativen Komplikation eines Enterothorax in Verbindung gebracht.

Methoden. In dieser aktuellen Publikation eines französischen High-volumeZentrums wurde der Einfluss der Hiatushernie auf den postoperativen Verlauf und das onkologische Outcome untersucht. Hierfür wurden die Daten von insgesamt 367 Patienten retrospektiv analysiert. Eingeschlossen wurden die Daten von Patienten mit der Diagnose eines Adenokarzinoms des distalen Ösophagus und gastroösophagealen Übergangs, die über einen transthorakalen Zugang ösophagektomiert wurden und bei denen der Hiatus oesophagei in der Computertomographie und in einer konventionellen Kontrastmitteluntersuchung dargestellt wurde. Die Autoren bildeten in Abhängigkeit von der Größe der Hiatushernie zwei Untersuchungsgruppen: 42 Patienten mit einer präoperativen Hia-
Ergebnisse. Die Autoren konnten zeigen, dass das mediane Überleben der 42 Patienten mit großer Hiatushernie signifikant schlechter war als in der Kontrollgruppe (20,9 vs. 41,0 Monate, $p<0,001$ ). Dieses Ergebnis wurde zum einen auf die deutliche geringere R0-Resektionsrate der Patienten mit Hiatushernie $>5 \mathrm{~cm}(50,0 \%$ vs. $4,0 \%, p<0,001)$, insbesondere am zirkumferenziellen Resektionsrand, zurückgeführt. Zum anderen war der Anteil der Patienten mit weit fortgeschrittener nodaler Metastasierung ( $\mathrm{pN} 3)$ in der Gruppe der großen Hiatushernien signifikant größer $(28,5 \%$ vs. $10,1 \%, p=$ 0,002 ). Die postoperative Morbidität war in beiden Gruppen bis auf die Rate der kardialen Komplikationen vergleichbar. Diese war bei Patienten mit Hiatushernie $<5 \mathrm{~cm}$ doppelt so hoch $(11,9 \%$ vs. $4,6 \%$, $p=0,05)$. Gleiches gilt für die 30-TageMortalität, die, wenn auch nicht signifikant erhöht, aber ebenfalls doppelt so hoch bei den Patienten mit großer Hiatushernie war $(7,1 \%$ vs. $3,1 \%, p=0,180)$.

Fazit. Dieses ist die erste, wenn auch retrospektive Analyse, die große Hiatushernien als Risikofaktor für ein schlechteres frühzeitiges und onkologisches Outcome identifizieren. Die Autoren erklären dieses Ergebnis damit, dass bei $\mathrm{Pa}$ tienten mit großen Hiatushernien ein Trend zu lokal fortgeschrittenen Tumoren nachzuweisen war und diese Tumoren schlechter auf die neoadjuvante Radiochemotherapie ansprachen. $\mathrm{Zu}$ Recht wird in der Diskussion mit externen Experten bei der hohen Rate inkomplet- ter zirkumferenzieller Tumorresektionen die Frage nach der chirurgischen Präparationstechnik des mediastinalen Peritonealsacks gestellt. Ob diese Daten zu einer Änderung des therapeutischen Konzeptes bei Diagnose einer großen Hiatushernie führen, kann gegenwärtig nicht beantwortet werden. Auch die Frage nach der operativen Versorgung der Hiatushernie im Rahmen der Ösophagektomie bleibt offen.

\section{Korrespondenzadresse}

Prof. Dr. W. Schröder, FACS, FEBS

Klinik für Allgemein-, Viszeral- und Tumorchirurgie, Universitätsklinik Köln Kerpener Str. 62, 50937 Köln, Deutschland wolfgang.schroeder@uni-koeln.de

Interessenkonflikt. W. Schröder und C. J. Bruns geben an, dass kein Interessenkonflikt besteht. 